\title{
ON THE SEMISIMPLICITY OF TWISTED GROUP ALGEBRAS
}

\section{S. PASSMAN}

ABstract. In this paper we make a number of simple observations on the question of the semisimplicity of twisted group algebras. The results are based for the most part on certain results known for algebraically closed fields and on the behavior of the Jacobson Radical under field extension.

Let $K$ be a field and let $G$ be a not necessarily finite group. We let $K^{t} G$ denote a twisted group ring of $G$ over $K$. That is, $K^{t} G$ is an associative $K$-algebra with basis $\{\bar{x} \mid x \in G\}$ and with multiplication defined by

$$
\bar{x} \bar{y}=\gamma(x, y) \overline{x y}, \quad \gamma(x, y) \in K-\{0\} .
$$

If all $\gamma(x, y)=1$ then $K^{t} G$ is in fact $K G$, the group ring of $G$ over $K$. In this paper we make a number of simple observations on the question of the semisimplicity of such algebras.

Theorem 1. Let $G$ be a group and set $\widetilde{G}=G \backslash Z$, the wreath product of $G$ by the infinite cyclic group $Z$. Then

(i) $G$ is isomorphic to a subnormal subgroup of $\tilde{G}$.

(ii) If $K$ is any field and if $K^{t} \widetilde{G}$ is any twisted group ring, then $K^{t} \tilde{G}$ is semisimple.

This result is implicit in [4] and [5] but it deserves more attention. It indicates that in general the question of semisimplicity is a global function of the group $G$. In particular it says that a knowledge of the structure of some subnormal subgroup of $G$ is never sufficient in itself to answer the question.

THEOREM 2. Let $K^{t} G$ be a twisted group ring and let $K_{0}$ be the subfield of $K$ generated by all $\gamma(x, y)$ with $x, y \in G$. Suppose that

(i) $K$ has characteristic $p>0$,

(ii) $G$ has no elements of order $p$, and

(iii) $K$ is not algebraic over $K_{0}$.

Then $K^{t} G$ is semisimple.

Corollary. Suppose that $K$ has characteristic $p>0$ and that $G$ has no elements of order $p$. If $K$ is not algebraic over $G F(p)$ then $K G$ is semisimple.

Received by the editors July 31, 1969.

AMS Subject Classifications. Primary 2080, 1630.

Key Words and Phrases. Twisted group algebras, semisimplicity, Jacobson Radical, field extensions. 
This improves Theorem IV of [3] by eliminating the separability condition required in that result. Thus we now know as much in characteristic $p>0$ about group rings of groups with no elements of order $p$ as we know (Theorem 1 of [2]) in characteristic 0 about group rings of arbitrary groups. On the other hand, for twisted group rings the characteristic 0 analog of Theorem 2 has not as yet been proved, so we in fact know more in the modular case.

THEOREM 3. Let $G$ be a solvable group and suppose that either $K$ has characteristic 0 or $K$ has characteristic $p>0$ and $G$ has no elements of order $p$. Then $K^{t} G$ is semisimple.

This is one of a number of similar results which can be proved by the same method, namely an inductive argument on the length of a finite normal series of $G$ provided that the quotient groups are of certain prescribed types. Presumably this method can be generalized and formalized as in [6].

1. Algebras. In this section we study the behavior of the Jacobson Radical of an algebra $A$ under field extension. This problem has of course been considered fully by Amitsur in [1]. However by making an appropriate assumption on $A$ we are able to circumvent to some extent the difficulty of inseparable field extensions.

In the following $A$ is a fixed algebra over $K, F$ and $L$ are field extensions of $K$ with $F \supseteq L \supseteq K$ and $A_{F}$ denotes the $F$-algebra $A \otimes_{K} F$. In addition $J(A)$ denotes the Jacobson Radical of $A$ and $N(A)$ denotes its Nilpotent Radical, so that $N(A)$ is the sum of all the nilpotent ideals of $A$.

Lemma 1.1. $J\left(A_{F}\right) \cap A_{L} \subseteq J\left(A_{L}\right)$.

Proof. Certainly $J\left(A_{F}\right) \cap A_{L}$ is an ideal in $A_{L}$. Let $\alpha \in J\left(A_{F}\right) \cap A_{L}$ so that $\alpha$ has a right quasi inverse $\beta \in A_{F}$. Let $\left\{f_{i}\right\}$ be a basis of $F / L$ with $f_{0}=1$. Then $A_{F}=\sum A_{L} \cdot f_{i}$ and we can write $\beta=\beta_{0}+\beta_{1}$ where $\beta_{0} \in A_{L}$ and $\beta_{1} \in \sum_{i \neq 0} A_{L} \cdot f_{i}$. From

$$
0=\alpha+\beta+\alpha \beta=\left(\alpha+\beta_{0}+\alpha \beta_{0}\right)+\left(\beta_{1}+\alpha \beta_{1}\right)
$$

we conclude that $\alpha+\beta_{0}+\alpha \beta_{0}=0$. Thus $\alpha$ has a right quasi inverse in $A_{L}$ so $J\left(A_{F}\right) \cap A_{L} \subseteq J\left(A_{L}\right)$.

LEMmA 1.2. Let $\mathfrak{F}_{0}$ be a family of fields such that

(i) $F \supseteq F_{0} \supseteq L$ for each $F_{0} \in \mathcal{F}_{0}$, and

(ii) for any finite subset $S$ of $F$ there exists $F_{0} \in \mathcal{F}_{0}$ with $S \subseteq F_{0}$. Let $\alpha \in A_{L}$ and suppose that $\alpha \in J\left(A_{F_{0}}\right)$ for all $F_{0} \in F_{0}$. Then $\alpha \in J\left(A_{F}\right)$.

Proof. Let $\beta \in A_{F}$. Then by (ii) $\beta \in A_{F_{0}}$ for some $F_{0} \in F_{0}$. Since 
$\alpha \in J\left(A_{F_{0}}\right)$ it follows that $\alpha \beta$ has a quasi inverse in $A_{F_{0}}$ and hence in $A_{F}$ by (i). Thus $\alpha A_{F}$ is a quasi regular right ideal and $\alpha \in J\left(A_{F}\right)$.

Proposition 1.3. Let $(F: L)=n<\infty$. Then $J\left(A_{F}\right)^{n} \subseteq J\left(A_{L}\right) \cdot A_{F}$ $\subseteq J\left(A_{F}\right)$.

Proof. Let $M$ be an irreducible $A_{L}$-module and consider the $A_{F-}$ module $M_{F}=M \otimes_{A_{L}} A_{F}$. As an $A_{L}$-module $M_{F}$ is a direct sum of $n$ irreducible modules. Hence clearly as an $A_{F}$-module, $M_{F}$ has a composition series of length $\leqq n$. Thus $J\left(A_{F}\right)^{n}$ acts trivially on $M_{F}$.

Let $f_{1}, f_{2}, \cdots, f_{n}$ be a basis for $F / L$ with $f_{1}=1$. Let $\alpha \in J\left(A_{F}\right)^{n}$ with $\alpha=\sum \alpha_{i} f_{i}$ and $\alpha_{i} \in A_{L}$. Since $M_{F} \alpha=0$ and $M_{F}=\sum M \otimes f_{i}$ we see that

$$
0=(M \otimes 1) \alpha=\sum\left(M \alpha_{i}\right) \otimes f_{i} .
$$

Thus $M \alpha_{i}=0$ and since this is true for all such irreducible $M$ we have $\alpha_{i} \in J\left(A_{L}\right)$. Thus $\alpha \in J\left(A_{L}\right) \cdot A_{F}$ and hence $J\left(A_{F}\right)^{n} \subseteq J\left(A_{L}\right) \cdot A_{F}$.

Now let $M$ be an irreducible $A_{F}$-module and let $\mu \in M-\{0\}$ so that $M=\mu\left(A_{F}\right)$. If $f_{1}, f_{2}, \cdots, f_{n}$ are as above then $M=\sum\left(\mu f_{i}\right) A_{L}$ and $M$ is a finitely generated $A_{L}$-module. Set $M_{0}=M\left(J\left(A_{L}\right)\right)$. Since $F$ commutes with $A_{L}$ it follows that $M_{0}$ is an $A_{F}$-submodule of $M$. Thus either $M_{0}=M$ or $M_{0}=0$. However by Nakayama's Lemma, since $M$ is a finitely generated $A_{L}$-module, we cannot have $M=$ $M\left(J\left(A_{L}\right)\right)$. Thus $0=M\left(J\left(A_{L}\right)\right)$ and since this is true for all such irreducible $M$ we have $J\left(A_{L}\right) \subseteq J\left(A_{F}\right)$. Thus $J\left(A_{L}\right) \cdot A_{F} \subseteq J\left(A_{F}\right)$.

Corollary 1.4. Let $F / L$ be algebraic. Then $J\left(A_{L}\right) \cdot A_{F} \subseteq J\left(A_{F}\right)$.

Proof. Let $\mathcal{F}_{0}$ be the family of all fields $F_{0}$ with $F \supseteq F_{0} \supseteq L$ and $\left(F_{0}: L\right)<\infty$. Since $F / L$ is algebraic it follows that $\mathcal{F}_{0}$ satisfies the hypotheses of Lemma 1.2. Thus by Lemma 1.2 and Proposition 1.3 we have $J\left(A_{L}\right) \subseteq J\left(A_{F}\right)$ and therefore $J\left(A_{L}\right) \cdot A_{F} \subseteq J\left(A_{F}\right)$.

Proposition 1.5. Let $F / L$ be purely transcendental and $F \neq L$. Then

$$
J\left(A_{\boldsymbol{F}}\right)=\left[J\left(A_{\boldsymbol{F}}\right) \cap A_{L}\right] \cdot A_{\boldsymbol{F}}
$$

and $J\left(A_{F}\right) \cap A_{L}$ is a nil ideal in $A_{L}$.

Proof. This is Theorem II of [1].

Definition. We say $A$ is nilpotent free if for all $F \supseteq K$ we have $N\left(A_{F}\right)=0$.

Lemma 1.6. Let $A$ be nilpotent free. Then $J\left(A_{L}\right)=0$ implies $J\left(A_{F}\right)$ $=0$.

Proof. Let $F_{0}$ be a subfield of $F$ with $F \supseteq F_{0} \supseteq L, F_{0} / L$ purely transcendental and $F / F_{0}$ algebraic. By Proposition 1.5, $J\left(A_{F_{0}}\right)=0$. 
Let $\alpha \in J\left(A_{F}\right)$. Since $F / F_{0}$ is algebraic it follows that there exists a field $F_{1}$ with $F \supseteq F_{1} \supseteq F_{0},\left(F_{1}: F_{0}\right)=n<\infty$ and $\alpha \in A_{F_{1}}$. Hence $\alpha \in J\left(A_{F}\right)$ $\cap A_{F_{1}} \subseteq J\left(A_{F_{1}}\right)$ by Lemma 1.1. By Proposition 1.3 we have $J\left(A_{F_{1}}\right)^{n}$ $\subseteq J\left(A_{F_{0}}\right) \cdot A_{F_{1}}=0$ and thus since $N\left(A_{F_{1}}\right)=0$ we have $J\left(A_{F_{1}}\right)=0$. This yields $\alpha=0$ and the result follows.

Corollary 1.7. Let $A$ be nilpotent free. If $F / L$ is algebraic then $J\left(A_{F}\right)=0$ if and only if $J\left(A_{L}\right)=0$.

Proof. By Lemma $1.6 J\left(A_{L}\right)=0$ implies that $J\left(A_{F}\right)=0$. On the other hand if $J\left(A_{L}\right) \neq 0$ then by Corollary $1.4 J\left(A_{L}\right) \subseteq J\left(A_{F}\right)$ so $J\left(A_{F}\right) \neq 0$.

Let $\operatorname{tr}(F / K)$ denote the transcendence degree of $F / K$ so that $\operatorname{tr}(F / K)$ is either a nonnegative integer or the symbol $\infty$.

Proposition 1.8. Let $A$ be nilpotent free and let $F$ and $F^{\prime}$ be two field extensions of $K$ with $\operatorname{tr}\left(F^{\prime} / K\right) \geqq \operatorname{tr}(F / K)$. Then $J\left(A_{F}\right)=0$ implies $J\left(A_{F^{\prime}}\right)=0$.

Proof. By Corollary 1.7 it suffices to assume that $F / K$ and $F^{\prime} / K$ are purely transcendental. By Proposition 1.5 it suffices to show that $J\left(A_{F^{\prime}}\right) \cap A=0$. Let $\alpha \in J\left(A_{F^{\prime}}\right) \cap A$ and let $\mathcal{F}_{0}$ be the set of all fields $F_{0}$ with $F \supseteq F_{0} \supseteq K$ and $F_{0} / K$ purely transcendental with finite transcendence degree. Since $F / K$ is purely transcendental it follows that $\mathcal{F}_{0}$ satisfies the hypotheses of Lemma 1.2. Let $F_{0} \in \mathcal{F}_{0}$. Since $\operatorname{tr}\left(F^{\prime} / K\right)$ $\geqq \operatorname{tr}(F / K)$ it follows that there exists a field $F_{0}^{\prime}$ with $F^{\prime} \supseteq F_{0}^{\prime} \supseteq K$ and such that $F_{0}$ and $F_{0}^{\prime}$ are $K$-isomorphic. Thus $A_{F_{0}}$ and $A_{F_{0}^{\prime}}$ are $A$ isomorphic. Now $\alpha \in J\left(A_{F^{\prime}}\right) \cap A_{F_{0}^{\prime}} \subseteq J\left(A_{F_{0}^{\prime}}\right)$ by Lemma 1.1 so it follows that $\alpha \in J\left(A_{F_{0}}\right)$. Since this is true for all such $F_{0} \in \mathcal{F}_{0}$, Lemma 1.2 yields $\alpha \in J\left(A_{F}\right)$ and hence $\alpha=0$. This completes the proof.

2. Group algebras. In this section $A=K^{t} G$, a twisted group ring of $G$ over $K$. If $F$ is a field extension of $K$ then clearly $A_{F}=F^{t} G$ with the same system $\{\gamma(x, y)\}$ of cofactors. In [5] a number of results were obtained about such algebras under the assumption that the field was algebraically closed of characteristic $p>0$. In most cases, in fact with the exception only of Theorem 3.2 of [5], a characteristic 0 analog of the result holds and is proved in the same way. In many cases the algebraically closed assumption is not needed either. In the following when we quote the results of [5] we will allow the field to have this more general nature.

Proof of Theorem 1 . Let $\bar{G}=G \backslash Z$. Then $G$ is clearly isomorphic to a subnormal subgroup of $\tilde{G}$. Let $F$ be the algebraic closure of $K$. We wish to show that $J\left(K^{t} \widetilde{G}\right)=0$ and by Corollary 1.4 it suffices to 
show that $J\left(F^{t} \tilde{G}\right)=0$. The result now follows from Proposition 6.3 of $[5]$.

For any prime $p$ let $\Delta^{p}(G)$ be the subgroup of $G$ generated by all elements $x \in G$ such that $x$ has order a power of $p$ and $\left|G: C_{G}(x)\right|$ $<\infty$.

Proposition 2.1. If $K$ has characteristic 0 then $K^{t} G$ is nilpotent free. If $K$ has characteristic $p>0$ then $K^{t} G$ is nilpotent free if and only if $\Delta^{p}(G)=\langle 1\rangle$.

Proof. Suppose that $K^{t} G$ is not nilpotent free and let $L$ be a field extension of $K$ with $N\left(L^{t} G\right) \neq 0$. Then $L^{t} G$ has a nonzero nilpotent ideal $I$. If $F$ is the algebraic closure of $L$ then clearly $I \otimes_{L} F$ is a nonzero nilpotent ideal in $F^{t} G$ so $N\left(F^{t} G\right) \neq 0$. By Theorem 3.7 of [5], $F$ has characteristic $p>0$ and $\Delta^{p}(G) \neq\langle 1\rangle$.

Conversely suppose that $K$ has characteristic $p>0$ and that $\Delta^{p}(G)$ $\neq\langle 1\rangle$. Let $F$ be the algebraic closure of $K$. Then by Theorem 3.7 of [5], $N\left(F^{t} G\right) \neq 0$ so $K^{t} G$ is not nilpotent free.

Proof of Theorem 2. Clearly $K^{t} G=K_{0}^{\imath} G \otimes_{K_{0}} K$ and since $G$ has no elements of order $p, K_{0}^{t} G$ is nilpotent free by Proposition 2.1. Let $F$ be a purely transcendental extension of $K_{0}$ with $\operatorname{tr}\left(F / K_{0}\right)=1$. By Proposition 1.5, $J\left(F^{t} G\right)=I \otimes F$ where $I$ is a nil ideal in $K_{0}^{t} G$. Thus by Theorem 3.2 of [5], whose proof does not require the field to be algebraically closed, we have $I=0$ and $J\left(F^{t} G\right)=0$. Now by assumption $\operatorname{tr}\left(K / K_{0}\right) \geqq \operatorname{tr}\left(F / K_{0}\right)$ so the result follows by Proposition 1.8. The corollary is of course an immediate consequence of Theorem 2 since if $K^{t} G=K G$ then $K_{0}=G F(p)$.

Lemma 2.2. Let $H \triangleleft G$ with $G / H=B$ abelian. Suppose that $J\left(K^{t} H\right)$ $=0$ and that $G$ has no elements of order $p$ if $K$ has characteristic $p>0$. Then $J\left(K^{t} G\right)=0$.

Proof. By Proposition 2.1, if $W$ is any subgroup of $G$ then $K^{t} W$ is nilpotent free. Let $F$ be the algebraic closure of $K$. Then by Corollary 1.7 we have $J\left(F^{t} H\right)=0$ and in addition it suffices to show that $J\left(F^{\prime} G\right)=0$. Let $\alpha \in J\left(F^{\prime} G\right)$. Then $\alpha \in F^{\prime} G_{0}$ where $G \supseteq G_{0} \supseteq H$ and $G_{0} / H$ is finitely generated. Hence also $\alpha \in J\left(F^{t} G_{0}\right)$ by Lemma 1.9 of [5]. It clearly suffices to show that $J\left(F^{t} G_{0}\right)=0$ or in other words we can assume that $G / H=B$ is a finitely generated abelian group.

Let $B_{1}$ be the torsion subgroup of $B$ so $B_{1}$ is finite of order $n$ for some integer $n$. Let $G \supseteq G_{1} \supseteq H$ with $G_{1} / H=B_{1}$. Now Proposition 1.3 of [5] yields $J\left(F^{t} G_{1}\right)^{n} \subseteq J\left(F^{t} H\right) \cdot F^{t} G_{1}=0$ and hence since $K^{t} G_{1}$ is nilpotent free we have $J\left(F^{t} G_{1}\right)=0$. Finally $G_{1} \triangleleft G$ and $G / G_{1}$ is a torsion 
free abelian group so $J\left(F^{t} G\right) \subseteq J\left(F^{t} G_{1}\right) \cdot F^{t} G=0$ by Corollary 1.11 of [5] and the result follows.

Proof of Theorem 3 . We proceed by induction on $d(G)$, the derived length of $G$. If $d(G)=0$ then $G=\langle 1\rangle$ and the result is clear. Now let $d(G)>0$. Then $d(G)>d\left(G^{\prime}\right)$, where $G^{\prime}$ is the commutator subgroup of $G$, so by induction $J\left(K^{t} G^{\prime}\right)=0$. Since $G / G^{\prime}$ is abelian, the above lemma yields $J\left(K^{\prime} G\right)=0$.

\section{REFERENCES}

1. S. A. Amitsur, The radical of field extensions, Bull. Res. Council Israel Sect. F 7F $(1957 / 58), 1-10$. MR 21 \#2675.

2. - On the semi-simplicity of group algebras, Michigan Math. J. 6 (1959), 251-253. MR $21 \# 7256$.

3. D. S. Passman, Nil ideals in group rings, Michigan Math. J. 9 (1962), 375-384. MR 26 \#2470.

4. - On the semisimplicity of modular group algebras, Proc. Amer. Math. Soc. 20 (1969), 515-519. MR 38 \#4576.

5. - - Radicals of twisted group rings, Proc. London Math. Soc. (to appear).

6. D. A. R. Wallace, Some applications of subnormality in groups in the study of group algebras, Math. Z. 108 (1968), 53-62.

Institute for Defense Analyses, Princeton, New Jersey 08540 and

UNiversity OF Wisconsin, MADISON, Wisconsin 53706 